\title{
Demonstrability, Difficulty and Persuasion: An Experimental Study of Advice Taking
}

\author{
Robert Hoffmann ${ }^{\text {a }}{ }^{\text {, Thomas Chesney }}{ }^{\mathrm{b}}$, Swee-Hoon Chuah ${ }^{\mathrm{c}}$, \\ Florian Kock ${ }^{\mathrm{d}}$, Jeremy Larner ${ }^{\mathrm{b}}$ \\ ${ }^{a}$ Behavioural Business Lab, School of Economics, Finance and Marketing, RMIT \\ University, Melbourne, Victoria 3000, Australia \\ ${ }^{\mathrm{b}}$ Nottingham University Business School, University of Nottingham, Nottingham \\ NG8 1BB, UK \\ ${ }^{\mathrm{c}}$ Tasmanian School of Business and Economics, University of Tasmania, Hobart, \\ Tasmania 7001, Australia

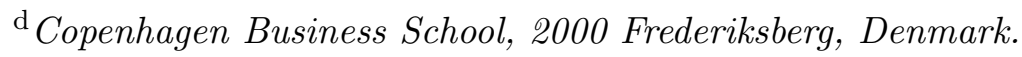

31 January 2020

\begin{abstract}
Self-interested paid advisors should try to sell their solutions no matter how they came about. However, we present evidence that advisor persuasiveness depends on two dimensions of their prior problem solving: solution difficulty and demonstrability. We report a laboratory experiment with repeated advisor-client interactions where both these dimensions are independently varied. Persuasion rises in solution demonstrability and falls in difficulty. The reason is non-optimising behaviour: Advisors lacking in confidence fail to conceal difficult problem solving and those receiving their advice baulk when the proposed solution lacks objective success criteria irrespective of its promise. Our findings suggest differential prospects for persuasion and selling of different kinds of products, services and ideas.
\end{abstract}

Key words: Persuasion; advisors; experiment; demonstrability; lying cost

\section{Introduction}

Good outcomes often entail the risk of relying on the decision making of better-informed advisors who may be biased (Wolinsky, 1993; Emons, 1997;

1 Corresponding author. Email: robert.hoffmann@rmit.edu.au. Funding from the UK Centre for Defence Enterprises (DSTLX1000046652) is gratefully acknowledged. We would like to thank Adrian Camilleri and Simon Gächter for suggestions and comments. 
Dulleck and Kerschbamer, 2006; Chakraborty and Harbaugh, 2007, 2010). In these situations, advisors first solve a decision problem and acquire private information and then persuade their clients of the correctness of their solution (e.g. Green and Stokey, 2007). These interactions are persuasion situations because advisors have motive and opportunity to mislead those who require their advice. Because of the ubiquity and importance of these kinds of situation, the underlying persuasion process between information sender (advisor) and receiver (client) is a central topic in different fields of social science.

We report the first experimental study of whether and how certain characteristics of the sender's decision problem affect the extent to which their advice is accepted. We explore two ways in which advisor choices may vary. First, Laughlin (1980) differentiates decision problem types along a spectrum of solution demonstrability. On one end, intellective decision problems (e.g. mathematical or lexical questions) have objectively appraisable solutions reached through a series of steps while judgment problems (e.g. ethical or aesthetic questions) typically lead to intuitive solutions which are harder to demonstrate to others. Second, advisor decision problems differ in how easy or difficult the correct solution is to determine (Pitchik and Schotter, 1987).

We examine whether and how the demonstrability and difficulty of decision problems independently affect information transmission and persuasion between sender and receiver. In standard theory, a rational and self-interested sender's advice should maximise her own payoffs irrespective of the nature of her decision problem or private information (Chakraborty and Harbaugh, 2007, 2010) as long as her communication constitutes cheap talk, i.e. is costless and unverifiable (Farrell and Rabin, 1996). However, in real settings, the nature of the sender's decision problem may affect persuasion through both sender and receiver behaviour. For the sender, more difficult tasks may reduce her confidence and, in turn, persuasion (e.g. Petty et al., 2002; Tenney et al., 2008; Sah et al., 2013). One reason is psychological disutility (lying costs), and another a pro-social regard for the receiver (Abeler et al., 2014; Lundquist et al., 2009).

Receiver behaviour may also be affected by decision problem characteristics. In real situations clients receiving advice are commonly aware of solution demonstrability and may temper their responses accordingly. This is because unlike difficulty, demonstrability is not a characteristic of a particular decision problem but a decision problem type to which any given problem may or may not belong. Low demonstrability requires greater trust by receivers to the extent that senders are unable to evidence the correctness of their answers.

In our sender-receiver experiment we vary demonstrability and difficulty independently and systematically and examine the effects on persuasion. The next section develops our theoretical base and motivation which we then illustrate using the example of venture capital (section 3). In section 4 we outline the 
experimental design, its implementation, followed by the variables we obtain and research hypotheses. Results are contained in section 5. We conclude and discuss policy implications in section 6 .

\section{Background}

\subsection{Persuasion}

Interpersonal persuasion is voluntary change in attitudes or behaviour of one individual that another intends through communication (Zimbardo and Leippe, 1991, p. 127). Most empirical studies in social psychology have used an experimental paradigm to measure attitude change in participants who receive persuasive messages (Ajzen 2012, p. 384; O'Keefe 2002, p. 23; ch. 7). The causal and moderating factors of attitude change identified in this literature include the motives and characteristics of both sender and receiver (Petty et al., 1997; Zimbardo and Leippe, 1991), of the content and medium of communication as well as of the situation (Janis et al., 1959; Cialdini, 1988).

In contrast, the persuasion literature in economics focusses on overt behaviour. A sender transmits private information relevant to a receiver's decision which determines both parties' payoffs. The question is to what extent receivers can glean useful information from a (rational) sender who communicates strategically through cheap talk (Farrell and Rabin, 1996; Crawford, 1998; van Winden, 1999). Studies have found that senders transmit more information than is rational (Cai and Wang, 2006) potentially due to lying aversion (SánchezPagés and Vorsatz, 2007) or the use of heuristics (Wang et al., 2010).

In both psychology and economics sender private information is generally perfect. However, in many realistic persuasion situations sender advice depends on solving a prior decision problem. For example, Green and Stokey (2007) study a two-person organisation where one is responsible for collecting information and the other for making decisions on its basis while their interests diverge. However there are no existing studies that examine how the nature of a sender's prior decision problem affects advice and its transmission.

\subsection{Demonstrability}

Laughlin and colleagues suggest that decision problems differ by the extent to which the correctness of their solutions can be evidenced (Laughlin, 1980; Laughlin and Ellis, 1986; Stasser and Stewart, 1992; Laughlin et al., 1995; Laughlin and Hollingshead, 1995; Laughlin and Shupe, 1996; Laughlin, 1999). Three factors contribute to solution demonstrability. One is the degree to which alternative solutions can be compared using a definitive and objective success criterion. The second is the extent to which the determination of the best solution involves a series of logical steps of reasoning (e.g. forensic evidence trails or clinical drug trials). The third factor is a shared conceptual 
(or epistemological) system within which both the solution success criterion and reasoning steps are established.

Problems in science and engineering generally possess objective criteria for solutions which can be arrived at through a series of steps within accepted methodology. In contrast, decision problems involving ethical or aesthetic judgments are examples of low solution demonstrability. Here the success of a solution lies in the eye of the beholder. It results from snap judgements and intuition rather than a series of logical steps. Moreover, judgments are subjective to the extent that the underlying moral or aesthetic norms are not universally shared.

Any decision problem can in principle be placed on a spectrum of demonstrability (Laughlin and Hollingshead, 1995; Laughlin and Shupe, 1996). Laughlin and Ellis (1986) find that the degree of demonstrability significantly facilitates agreement among decision group members. In the following we report the first application of demonstrability to the sender-receiver game literature. Our work also contributes to the demonstrability literature in that we examine it in dyadic (rather than group) interactions with asymmetric information and conflict of interest.

\subsection{Difficulty}

Most previous work assumes sender private information to be perfect, i.e. known to be correct with certainty. Instead we consider situations where private information is generated through imperfect sender decision making. For example, in Pitchik and Schotter's (1987) model of consumer advice, an advisor's competence is variable so that advice is incorrect with some probability. In many other realistic scenarios the quality of private information may be variable. Retailers and sales negotiators not involved in the production process often have only partial knowledge regarding product specifications and quality. Similarly, financial advisors cannot perfectly predict the future performance of different investment products. In these cases the sender acquires her private information through search subject to perception and processing errors leading to imperfect information.

Sender advice therefore varies in quality depending on her competence and the problem's characteristics. We are interested here in the latter, the inherent solvability of the problem itself. We define difficulty as the ex ante probability that a randomly-chosen decision maker will identify the correct solution. We examine whether increasing problem difficulty (and therefore decreasing information quality) will lead to less persuasion in practice. In theory, unless the situation is repeated (Golosov et al., 2014), any sender information quality is irrelevant to the game's outcome since cheap talking senders have an incentive to exaggerate (e.g. Chakraborty and Harbaugh, 2010). However, with greater difficulty, senders may exploit private information less due to altruism, 
self consciousness, reputation or ethical principle creating a kind of curse of knowledge (see Camerer et al., 1989, p. 1244). In this sense, talk is not cheap because deception entails a psychic lying cost (Abeler et al., 2014).

\section{An illustration: Venture capital}

Venture capital illustrates how demonstrability and difficulty affect persuasion. Entrepreneurs identify business opportunities and solutions for their exploitation that require funding from venture capitalists. This interaction is characterised by both asymmetric information and misaligned interests: Entrepreneurs have greater knowledge of the opportunity and the incentive to maximise outside investment while venture capitalists lose from investing in unsuccessful projects (Martens et al., 2007; Carpentier and Suret, 2015; van Werven et al., 2015). Venture capitalists must glean useful information (and disregard misinformation) from the entrepreneur's storytelling or "pitching" to persuade them (de Bettignies and Brander, 2007; Pollack and Bosse, 2014; Martens et al., 2007; Herzenstein et al., 2011).

In terms of the illustration we examine whether investment depends on the nature of the venture creation decision problem. Venture creation situations differ both in how accurately business opportunities can be identified, assessed and developed, and how easily their prospects can be demonstrated. On one end of the demonstrability spectrum there are projects that can be evaluated in a series of steps according to objective criteria such as sales forecasts or technical feasibility studies for new products. In the pharmaceutical and natural resource extraction industries, the prospects of particular projects can often be ascertained and documented with reference to research (e.g. clinical trial data and geophysical surveys).

On the other end, projects in the creative and aesthetic realms, such as entertainment production or fashion, lack objective criteria but depend on judgment to anticipate the subjective aesthetic evaluations of others. For example, due to uncertain market and demand conditions, movie making is increasingly financed by venture capital investment based on the vision and competence of artistic entrepreneurs that determine success (DeFillippi and Arthur, 1998). The combination of uncertain public reception and highly specific individual competence make the prospects of movie projects hard to demonstrate.

Consumer electronics represent an industry in the middle of the demonstrability scale because of a mixture of technical performance features that can be demonstrated, and aesthetic ones that cannot (e.g. Apple's Power Mac G4 Cube, Linzmayer, 2004, p. 299). Another example is that of project-based professional services (software, financial, legal and management consulting). While performance criteria (such as previous sales and returns, courtroom success or subsequent performance of the consulted firm) can be documented, 


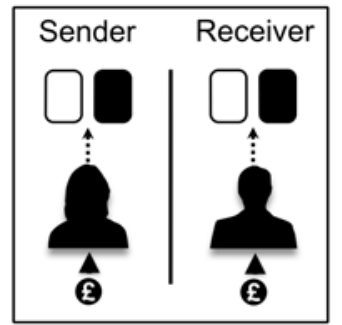

Phase A task

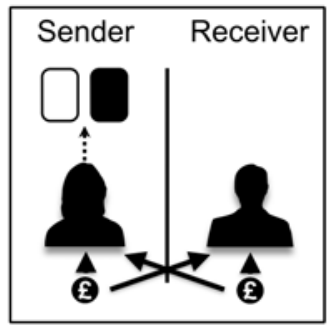

Phase B task

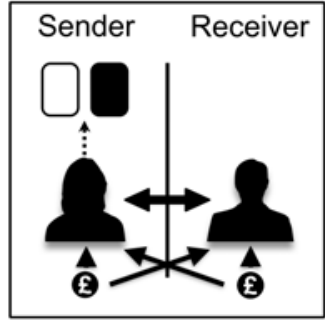

Phase C task

Fig. 1. Schematic illustration of the experimental tasks in the three phases.

the idiosyncrasy of projects and the importance of individual personnel reduce their cogency.

Venture creation projects also differ in terms of the difficulty of assessing their prospects. Oil and gas exploration projects may be assessed more or less easily depending on the nature and location of particular natural resource deposits. New consumer product technology may involve either breakthrough or marginal innovation. Artistic projects such as movies or music talent spotting may uncover obvious and unequivocal or more risky prospects.

\section{Experiment}

We conducted an experiment to examine the effects of demonstrability and difficulty on the persuasiveness of senders.

\subsection{Phases}

Participants completed experimental tasks in three phases (see Figure 1) where the first two (A and B) serve as controls and preparation for the proper measurement of persuasion in phase $\mathrm{C}$.

In phase $\mathrm{C}$ (communication phase) each sender is matched with every receiver for a total of 9 interactions using round-robin matching (every sender with every receiver in the experimental session). In each such interaction the sender is presented with a pair of images and asked to identify the correct one in response to a true-or-false question. The sender then decides any part of 100 points to invest in her answer. The receiver observes the question the sender must answer but not sender decision, the amount invested or the actual images shown. Following the sender decisions there is a fixed period of unrestricted 2-way communication between sender and receiver. Next, the receiver decides which part of a stake of 100 points to invest in the sender's image answer. Note in this design the decision problem is given only to the sender not the receiver, unlike the Laughlin group decision studies. The payoffs for each sender and each receiver respectively are their own uninvested points plus 1.5 times their invested points if the sender's image answer was correct. All invested points are forfeited if the sender's answer is incorrect. Both sender and receiver are 
also given the other's earnings from the invested points. ${ }^{2}$

Consider an illustration. The sender is shown the Mickey Mouse image pair in figure 2 and answers the question, "Which is more black?" with the one on the right, and then decides to invests 50 points. The sender then communicates with the receiver who then invests 20 points in the sender's answer which turns out to be correct. The sender receives $50+(50 \times 1.5)=125$ points from her own decision plus an additional $(20 \times 1.5)=30$ based on the receiver's investment for a total of 155 . The receiver earns $80+(20 \times 1.5)=110$ from his own decision plus $50 \times 1.5=75$ from the sender's investment decision for a total of 185. Had the sender's answer been incorrect her earnings would have been $(50+50 \times 0)+(20 \times 0)=50$. The receiver would have earned $(80+20 \times 0)+(50 \times 0)=80$.

The logic of this task is as follows. Because the sender receives any earnings the receiver makes from investing in the sender's image answer, the sender has an incentive to communicate so to persuade the receiver to invest the maximum irrespective of the sender's own confidence in her image answer. The receiver (who cannot change the investment decision already made by the sender) may benefit from communication only by correctly gleaning the likelihood of a correct image answer from the sender and invest accordingly. ${ }^{3}$ The receiver's investment reflects, to an extent, the degree of persuasion.

Phase $\mathrm{C}$ was preceded by two additional phases (Figure 1): first, A (accustomisation), followed by B (blind). Phase B proceeded in exactly the same fashion as Phase $\mathrm{C}$ except that there was no possibility of communication. Further there were only three image pairs, one of each type. The rationale for Phase $\mathrm{B}$ is that receiver investment in Phase $\mathrm{C}$ may be motivated by factors beyond communication with the sender, such as receiver risk appetite. Phase B generates observations we use to control for these factors. In addition, there was a Phase $A$ in which both senders and receivers see and invest in 9 image pairs and receive earnings only from their own decisions without any communication between them. The purpose was to allow senders as well as receivers to familiarise themselves with the image tasks (and their own accuracy) before performing investments in sender image decisions in phases B and C. Every participant was shown different image pairs in every interaction in Phases A and $\mathrm{B}$ which, as in Phase C, differed in terms of demonstrability as well as difficulty. These pairs were also different to the ones shown in Phase C: No participant saw the same image pair more than once in the experiment.

\footnotetext{
2 For senders this feature provides the incentive to pursuade. For receivers it has been added in the interest of symmetry, i.e. to avoid potential effects on investment decisions from envy or guilt arising from unequal opportunities for payoffs (e.g. Kirchsteiger, 1994; Jordan et al., 2015).

3 The task is a mixed-motive (non-zero-sum) game to the extent that motives increasignly overlap with the (uncertain) degree of sender accuracy.
} 


\subsection{Treatments}

Nine different image pairs were used in Phase $\mathrm{C}$ in a $3 \times 3$ design to manipulate our two treatment variables demonstrability and difficulty are shown in Figure 2. Demonstrability was varied three ways using three kinds of image task based on Laughlin (1980). For skill, the highest demonstrability condition, we used perceptual discrimination tasks. Participants were asked to indicate which of the two pure black and white images (one a colour inversion of the other) had more black pixels. The answer is in principle demonstrable to the extent that it can be derived by a series of operations (Laughlin, 1980), e.g. by dividing the image into equal-sized squares and counting the balance of black to white ones. For knowledge, our intermediate level of demonstrability, we used a semantic memory task. Each of the two images showed a different photograph of an iconic international landmark and participants were asked which was in a particular country. So-called world knowledge problems such as this lie in the middle of the demonstrability spectrum (Laughlin, 2011, pp. 93, 110). While there is a single true-or-false success criterion, the demonstrability of correct answers here is limited by the extent to which senders can accurately convey the contents of the two images, and their reasons for determining the correct answer. An aesthetic evaluation task was used for the lowest level of demonstrability (judgment). Two photographs of young people of the same gender and ethnicity were presented, sourced from a public rating website. The question was which was rated as more attractive on the site. This task is at the judgment end of demonstrability spectrum as the consensus of an external group of judges is considered least demonstrable (Laughlin, 1980). The 9 Phase $\mathrm{C}$ image pairs were presented to every sender in the order indicated by the numbered boxes in Figure 2. In all phases skill image pairs were shown first, followed by knowledge and finally judgment to better reflect realistic scenarios. This ordering as well as presenting receivers with the precise question (but not the actual image pairs) senders were given means receivers were aware of demonstrability. Receivers in real advisor persuasion situations (such as sales pitches) ordinarily know demonstrability precisely because solutions are sold to them. However this is not true for difficulty. Within each level of demonstrability, there was no ordering of the three pairs by their difficulty. All images in all phases were presented in the same order to every sender.

\subsection{Procedure}

We recruited 234 undergraduate student participants at a large UK university from different faculties via class announcements and posters. Exactly half (117) were male and the average age was 21.3 years. There were 13 sessions with 18 participants in each. Upon arrival, participants were equally and randomly split between two separate laboratory rooms, one for senders and the other for receivers. Laboratories were equipped with partitioned computer terminals running z-Tree (Fischbacher, 2007) to present tasks, elicit responses, administer 


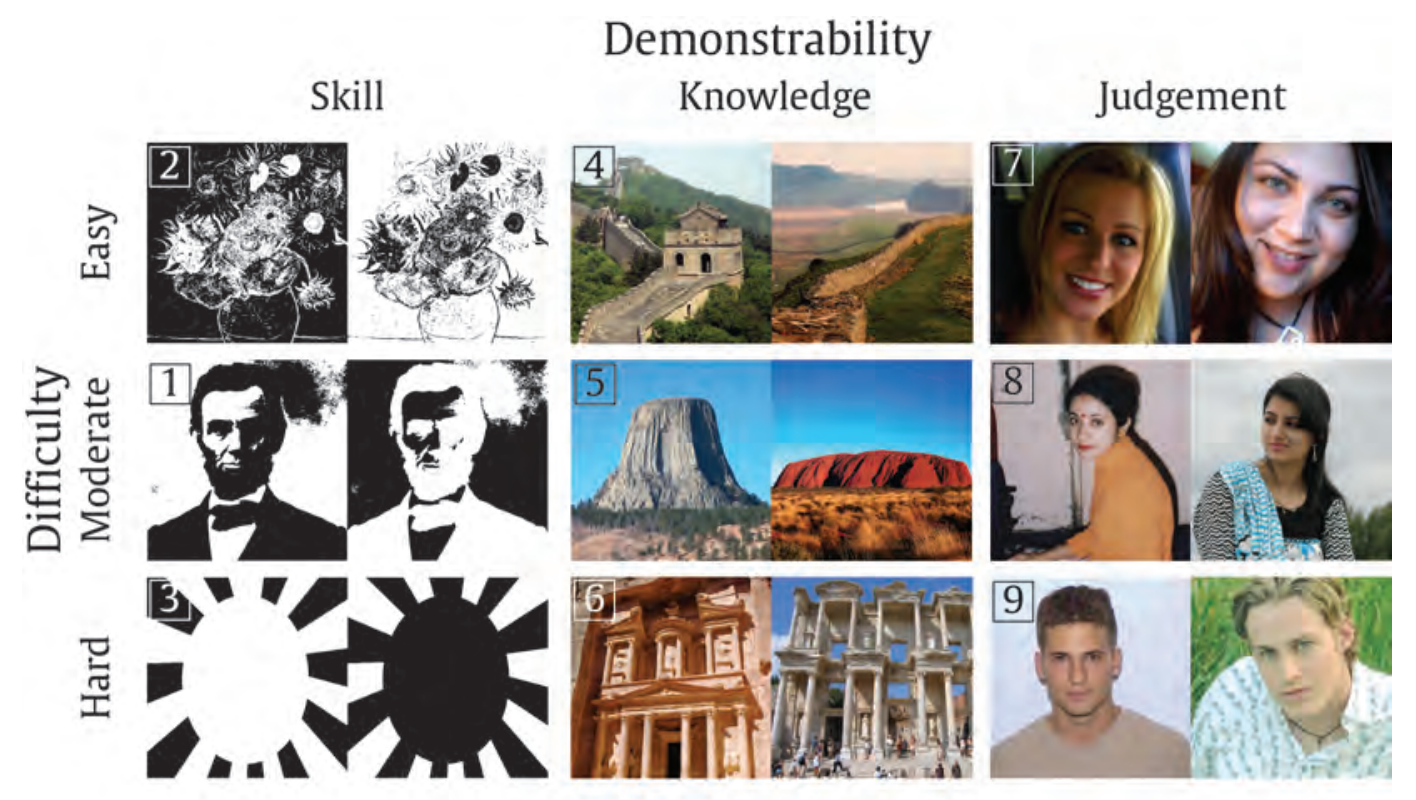

Fig. 2. The nine image pairs used as Phase $\mathrm{C}$ tasks in the $3 \times 3$ design. The image of a cartoon mouse that appeared to participants in pair 3 is not shown here for copyright reasons.

comprehension quizzes and questionnaire responses, match participants, facilitate communication, provide feedback and calculate earnings. In particular, communication between senders and receivers in phase $\mathrm{C}$ was an unrestricted 90 seconds of 2-way communication using z-Tree's instant message feature immediately after the sender investment decision. Sessions lasted around 90 minutes. We maintained a controlled experimental environment throughout including privacy.

The three phases A, B and C proceeded in that order after consent form completion and general announcements. Every phase began with paper instructions and a compulsory comprehension quiz and ended with individual feedback on performance for each task in the phase. No feedback was given immediately after each individual image tasks. We did not use deception in the experiment. All payoff-relevant parts of the experiment including payment scheme were made common knowledge among all participants. After phase $\mathrm{C}$ participants completed a questionnaire with demographic questions. As the investment task involves a dimension of risk we also elicited participants' "willingness to take risks, in general" on an eleven-point scale, a measure that has been shown to predict experimental behaviour by Dohmen et al. (2011).

Participants knew that the experiment was conducted under conditions of incentive compatibility, i.e. performance-related pay (e.g. Croson, 2005). At the end of the session participant earnings were determined as the total number of points earned over three tasks converted at the rate of $£ 0.4$ per 100 points plus a flat participation fee of $£ 10$. On average participants were paid out around 
$£ 23$ (maximum: 27, minimum: 20) in cash delivered privately immediately after the session. ${ }^{4}$

\subsection{Variables and hypotheses}

Our experiment generated a dataset with observations concerning 1053 senderreceiver games in phase C, i.e. 9 senders being matched with each of 9 receivers in every one of 13 sessions. Experimental variables are shown in table 1. At the level of each game, we observe whether the sender's decision is accurate ( $\mathrm{SACC}=0$ or 1 ), the time in seconds the image selection decision took (STIME), the total points both sender and receiver invest (between 0 and 100) in the sender's image answer (SINV and RINV). SINV is an incentive-compatible measure of the confidence a sender has in her decision. RINV is our main dependent variable as it constitutes the target of sender persuasion attempts. Because RINV in phase $\mathrm{C}$ reflects both the effect of communication and receiver-specific motives to invest we derive a measure of persuasion (PERS) as the difference between RINV in phase $\mathrm{C}$ and RINV in phase B (BLIND RINV). The latter variable is, for a given interaction, the average of what the sender concerned invested into images of the same image type in phase B. For the same reason we use a dummy variable to indicate phase $\mathrm{C}(\mathrm{COMM}=1$ else 0 ). We also examined chat logs and recorded observations for 505 games where senders made claims about SINV, the amount they invested (SCLAIM). ${ }^{5}$ We created ordinal variables for the difficulty treatment $(\mathrm{DIFF}=1$ for easy, $=2$ for moderate and $=3$ for hard) as well as for demonstrability (DEMO $=3$ for skill, $=2$ for knowledge and $=1$ for judgment). SKILL, KNOW and JUDGE are separate dummy variables for each of these three levels of demonstrability.

The central proposition of this paper is that persuasion is positively related to demonstrability, and negatively to difficulty. Our hypotheses for the relationships between these variables are based on the following conceptual framework (figure 3). Persuasion is the result of the interaction between sender and receiver based on the sender's prior decision problem which is characterised by demonstrability and difficulty. The sender's confidence in her solution, proxied by SINV results from difficulty of the decision problem alone and not its demonstrability (H1).

Hypothesis 1 Difficulty has a negative effect on SINV, however, demonstrability does not affect SINV.

In the subsequent interaction, communication from the sender results in persuasion of the receiver. One important component of the communication that we measure is the amount the sender claims to have invested. While rational and income maximising senders will exaggerate their own investments, this

\footnotetext{
$\overline{4}$ At the time of the experiment £1 Sterling (GBP) traded at 1.51 USD.

5 In 12 of the 505 games senders under-claimed their investments, i.e. SINV $>$ SCLAIM. We did not use these data.
} 
Table 1

Experimental variables.

\begin{tabular}{llrrrrrr}
\hline Variable & Phase & Obs & Mean & Std Dev & Min & Max & Range \\
\hline SINV & C & 1053 & 72.59 & 29.5 & 0 & 100 & $\{0 \ldots 100\}$ \\
STIME & C & 1050 & 8.98 & 6.1 & 0 & 20 & \\
SACC & C & 1053 & 0.79 & 0.4 & 0 & 1 & $\{0,1\}$ \\
SCLAIM & C & 505 & 79.15 & 26.9 & 0 & 100 & \\
RINV & C & 1053 & 66.84 & 34.3 & 0 & 100 & $\{0 \ldots 100\}$ \\
BLIND RINV & B & 351 & 51.56 & 32.4 & 0 & 100 & $\{0 \ldots 100\}$ \\
PERS & B and C & 1053 & 15.28 & 39.0 & -100 & 100 & $\{-100 \ldots 100\}$ \\
\hline & & Treatment variables & & & \\
\hline COMM & B and C & 1404 & 0.75 & 0.4 & 0 & 1 & $\{0,1\}$ \\
DEMO & C & 1053 & 2.00 & 0.8 & 1 & 3 & $\{1,2,3\}$ \\
SKILL & C & 1053 & 0.33 & 0.5 & 0 & 1 & $\{0,1\}$ \\
KNOW & C & 1053 & 0.33 & 0.5 & 0 & 1 & $\{0,1\}$ \\
JUDGE & C & 1053 & 0.33 & 0.5 & 0 & 1 & $\{0,1\}$ \\
DIFF & C & 1053 & 2.00 & 0.8 & 1 & 3 & $\{1,2,3\}$ \\
\hline
\end{tabular}

Sender decision problem

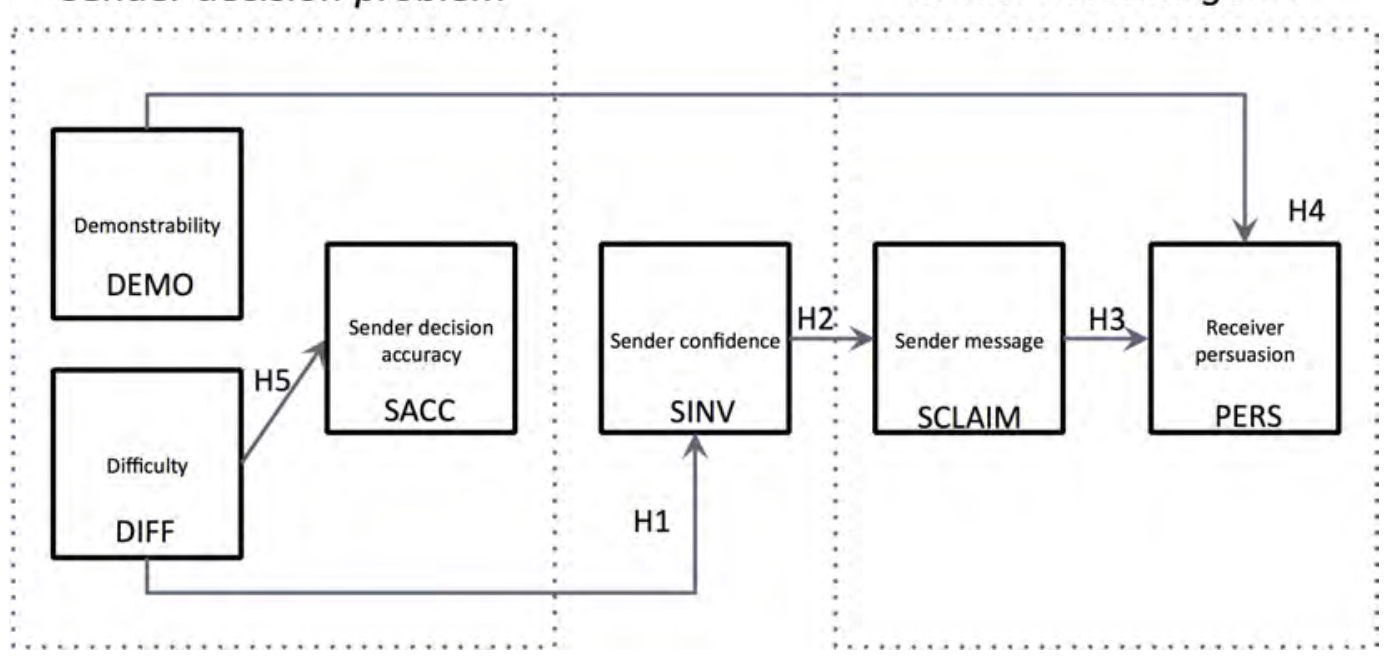

Fig. 3. Conceptual model with experimental variables and research hypotheses. 
amount mirrors real investments to the extent that senders are honest (H2). A sender with sufficiently strong aversion to lying or with pro-social preferences may report SINV correctly. Difficulty therefore affects persuasion through sender confidence and message. Note that because senders do not know their own accuracy, SACC is not hypothesised to influence either SINV or SCLAIM. Feedback was provided only after the task.

Hypothesis 2 SINV has a positive effect on SCLAIM.

RINV reflects persuasion in the extent to which a receiver's investment decision is are influenced by senders' cheap talk. A receiver's decision to invest is influenced by the sender's claim (H3). Further, because receivers know demonstrability independently, its level positively affects the receiver's decision to invest to the extent that receivers are reluctant to take solutions that cannot be evidenced on trust (H4).

Hypothesis 3 SCLAIM has a positive effect on PERS.

Hypothesis 4 DEMO has a positive effect on PERS.

Finally, sender decision accuracy depends on difficulty but not demonstrability (H5). Harder decision are less likely to a yield successful solution irrespective of demonstrability which does not affect the sender's ability to find it.

Hypothesis 5 DIFF, but not DEMO, has a negative effect on SACC.

\section{Results}

\subsection{Participant Communication}

Our hypotheses concern the effects of two main independent variables, DIFF and DEMO, on persuasion. We begin by examining the text chat transcripts for evidence that the difficulty of the senders' decision problems and the demonstrability of the correctness of their solutions indeed featured in the persuasion process as expressed in their communications.

Senders and receivers each typically sent between 5 and 10 messages during their 90-second exchange. Apart from banter, conversations were mostly information exchange relevant to the task. Typically receivers asked questions that senders responded to. These questions were mostly about task difficulty and demonstrating solution correctness. For difficulty, most receivers asked senders how difficult they thought the task was, how certain they were about their answers and how much they invested. In terms of demonstrability, receivers tended to ask about the images' types, descriptions of the image particulars, and the senders' solution processes. Senders often described the images, how they arrived at their solutions and what objective criteria they used.

An exchange between two participants that contains these elements is shown in 


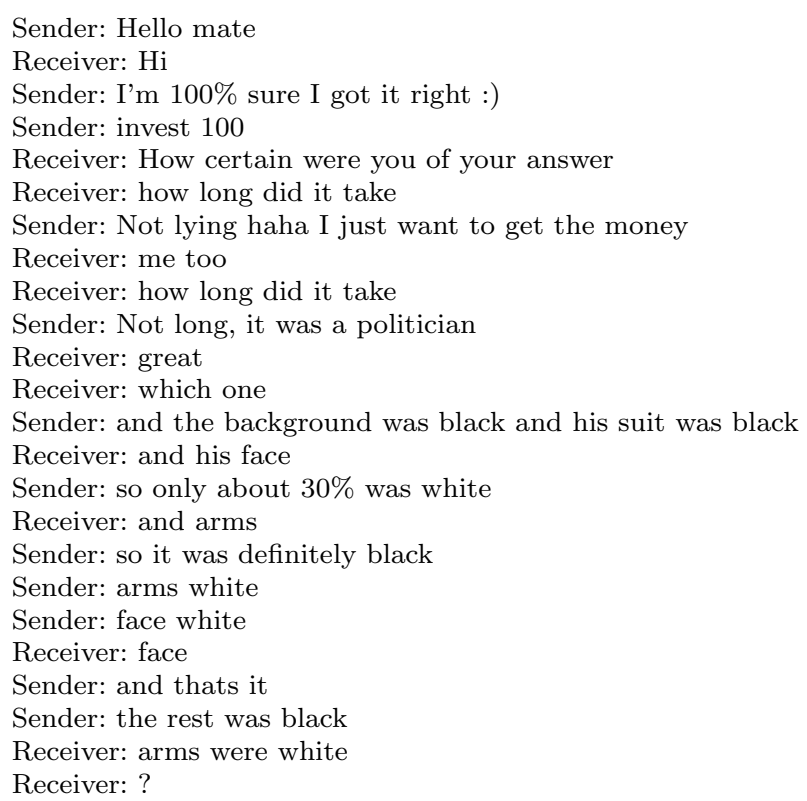

Fig. 4. Text message exchange between two participants. The task is the Abraham Lincoln pair shown in figure 2 .

figure 4. After claiming complete confidence, the receiver deconstructs the image of Abraham Lincoln into separate elements (background, suit), a procedure that is continued by the receiver (face, arms) resulting in an overall estimation of the black-white balance. As discussed, the determination of a solution using logical steps (the number and size of different elements of a picture) is one aspect of solution demonstrability. Other aspects of demonstrability were used for the other image types. For example, many senders attempted to invoke objective criteria to the low-demonstrability beauty task. These included resemblance to famous people, blond hair and blue eyes and perceived health of the models that senders judged more attractive.

It should be noted that there was considerable variation in both the length and nature of sender-receiver exchanges, which exhibited these different persuasive appeals and questions to different extents. However, we interpret these findings to support that both difficulty and demonstrability were used by participants in the persuasion process.

\subsection{Regression Results}

We now turn to the analysis of the data from the experiment. Summary statistics for our variables are shown in table 1 . The distributions of behavioural variables over the experimental conditions are displayed in figure 5. Our focus is the effect of demonstrability and difficulty on the persuasion process captured by SACC, SINV, SCLAIM and RINV according to our hypotheses and conceptual model (see figure 3). 


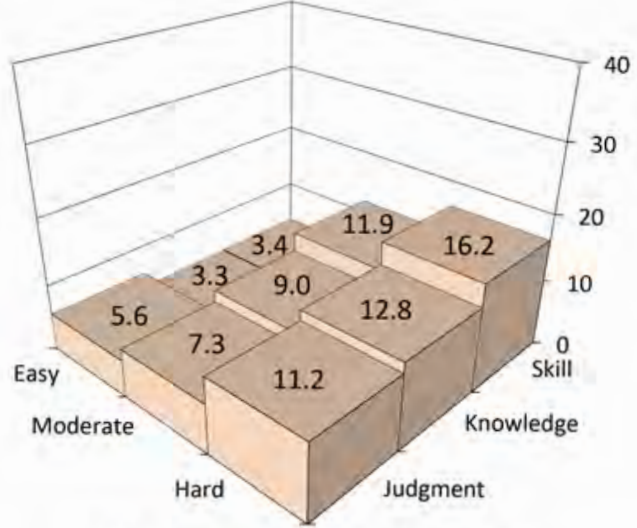

STIME (obs=1050, mean=9.0, st. dev=6.1)

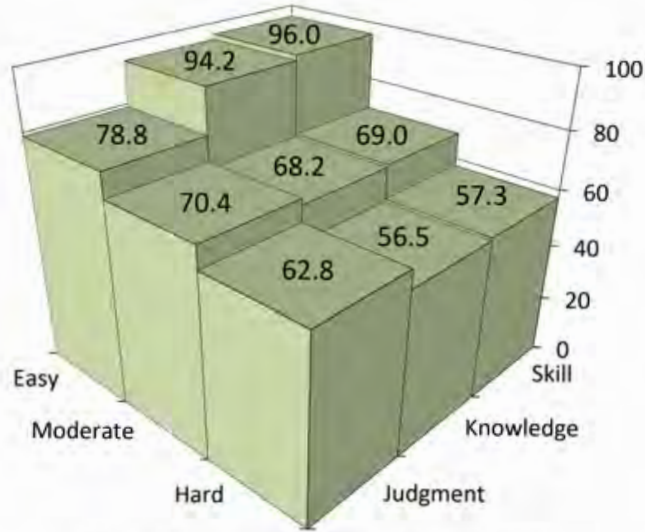

SINV (obs=1053, mean=72.6, st. dev=29.5)

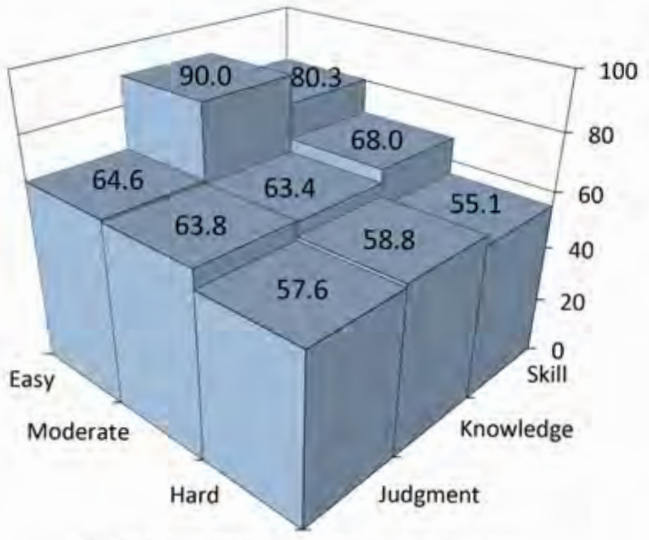

RINV (obs=1053, mean=66.8, st. dev=34.3)

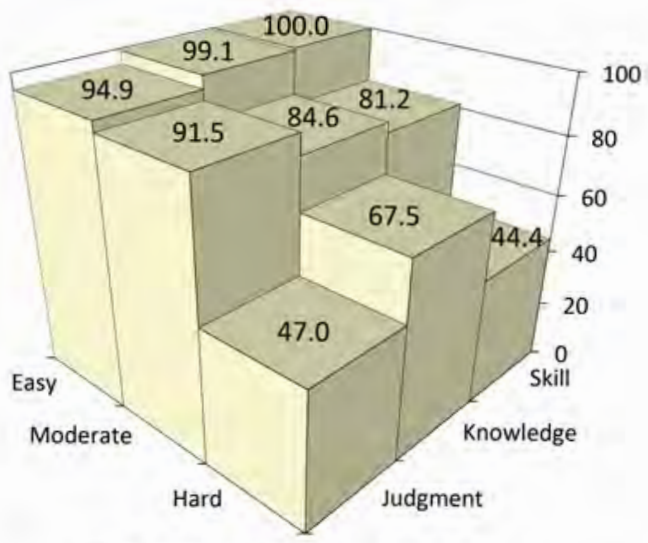

SACC (obs=1053, mean $=78.9$, st. $\operatorname{dev}=40.1$ )

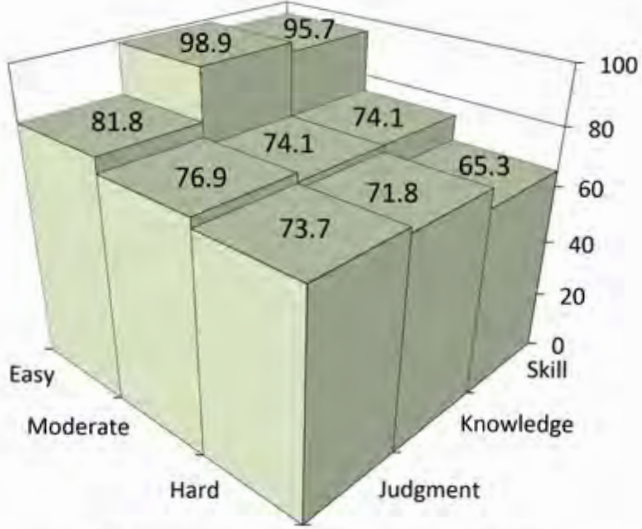

SCLAIM (obs=505, mean=79.1, st. dev=26.9)

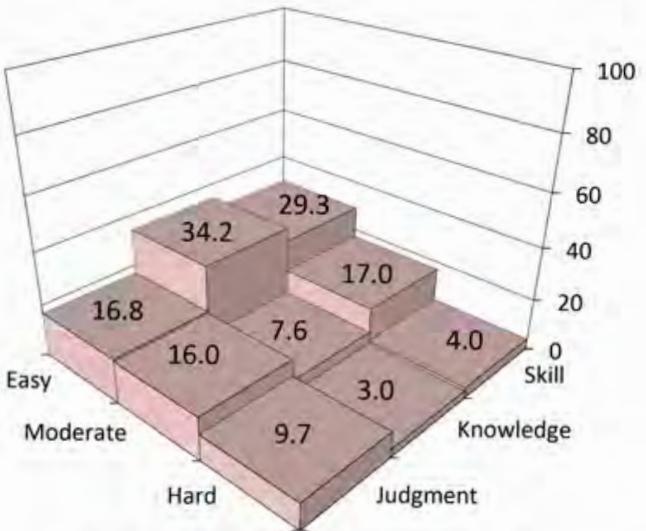

PERS (obs $=1053$, mean $=15.3$, st. $\operatorname{dev}=39.0$ )

Fig. 5. Distribution of behavioural variables (as averages) over levels of demonstrability (skill, knowledge and judgment) and difficulty (easy, moderate and hard).

We first analyse our data using a standard regression approach. Results are presented in table 5.2. Because of repeated observations for individual participants we use a participant-level random-effects approach. We only use data from phase $\mathrm{C}$ for all variables but BLIND RINV. We start by examining the effect of difficulty on the sender's decision (H 5). Regression model 1 supports 
Table 2

Regression results for SACC, SINV, SCLAIM and RINV. Standard errors in paren-

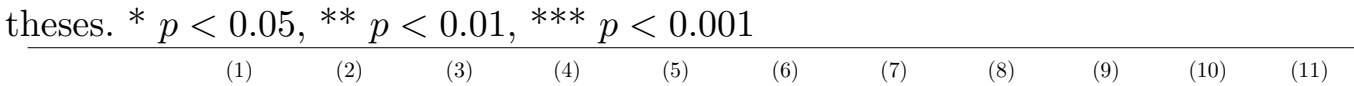

\begin{tabular}{|c|c|c|c|c|c|c|c|c|c|c|c|}
\hline DV: & SACC & SACC & SINV & SINV & SCLAIM & SCLAIM & RINV & RINV & RINV & RINV & RINV \\
\hline \multirow[t]{2}{*}{ DIFF } & $-1.783^{* * *}$ & $-1.784^{* * *}$ & $-15.41^{* * *}$ & $-15.41^{* * *}$ & $-10.80^{* * *}$ & -0.00949 & $-10.59^{* * *}$ & $-10.59^{* * *}$ & $-3.968^{* *}$ & $-3.987^{* *}$ & $-3.879^{* *}$ \\
\hline & $(0.141)$ & $(0.141)$ & $(1.001)$ & $(1.001)$ & $(1.363)$ & $(1.043)$ & (1.029) & (1.024) & $(1.288)$ & $(1.281)$ & $(1.357)$ \\
\hline \multirow[t]{2}{*}{ DEMO } & -0.0991 & -0.0991 & 1.721 & & 0.456 & -1.393 & $2.893^{* *}$ & & $3.201^{* *}$ & & $3.176^{* *}$ \\
\hline & $(0.105)$ & $(0.105)$ & $(1.001)$ & & (1.353) & $(0.936)$ & (1.029) & & $(1.201)$ & & $(1.212)$ \\
\hline \multirow[t]{2}{*}{ SESSION } & & 0.00682 & & & & & & & & & \\
\hline & & $(0.00982)$ & & & & & & & & & \\
\hline \multirow[t]{2}{*}{ KNOW } & & & & 2.276 & & & & $8.704^{* * *}$ & & $7.884^{* *}$ & \\
\hline & & & & $(2.003)$ & & & & $(2.047)$ & & $(2.437)$ & \\
\hline \multirow[t]{2}{*}{ SKILL } & & & & 3.442 & & & & $5.786^{* *}$ & & $6.318^{* *}$ & \\
\hline & & & & $(2.003)$ & & & & $(2.047)$ & & $(2.389)$ & \\
\hline \multirow[t]{2}{*}{ SINV } & & & & & & $0.664^{* * *}$ & & & & & 0.0111 \\
\hline & & & & & & $(0.0279)$ & & & & & $(0.0548)$ \\
\hline \multirow[t]{2}{*}{ BLIND RINV } & & & & & & & $0.429^{* * *}$ & $0.429^{* * *}$ & $0.461^{* * *}$ & $0.458^{* * *}$ & $0.461^{* * *}$ \\
\hline & & & & & & & $(0.0642)$ & $(0.0642)$ & $(0.0836)$ & $(0.0842)$ & $(0.0821)$ \\
\hline \multirow[t]{2}{*}{ SCLAIM } & & & & & & & & & $0.663^{* * *}$ & $0.657^{* * *}$ & $0.655^{* * *}$ \\
\hline & & & & & & & & & $(0.0414)$ & $(0.0413)$ & $(0.0602)$ \\
\hline \multirow[t]{2}{*}{ Constant } & $5.648^{* * *}$ & $5.553^{* * *}$ & $99.98^{* * *}$ & $101.5^{* * *}$ & $99.83^{* * *}$ & $34.68^{* * *}$ & $60.10^{* * *}$ & $61.05^{* * *}$ & -10.71 & -8.222 & -10.94 \\
\hline & $(0.435)$ & $(0.454)$ & $(2.955)$ & $(2.463)$ & $(4.031)$ & $(3.896)$ & $(4.706)$ & $(4.390)$ & $(7.018)$ & $(6.739)$ & $(7.063)$ \\
\hline$N$ & 1053 & 1053 & 1053 & 1053 & 505 & 505 & 1053 & 1053 & 505 & 505 & 505 \\
\hline$l l$ & -420.6 & -420.4 & & & & & & & & & \\
\hline$\chi^{2}$ & 160.3 & 160.5 & 240.1 & 240.0 & 62.94 & 697.9 & 158.5 & 170.4 & 384.5 & 392.2 & 384.0 \\
\hline$R^{2}$ & & & 0.185 & 0.185 & 0.110 & 0.582 & 0.172 & 0.178 & 0.438 & 0.442 & 0.438 \\
\hline
\end{tabular}

this hypothesis in that more difficult images significantly cause less accurate sender decisions that while demonstrability had no effect on accuracy. Model 2 includes an index for the timing of the experimental session to test whether participant collusion between sessions could have affected their ability to solve the image tasks. This variable is insignificant suggesting there was no effect of collusion on accuracy. Similar to model 1, in model 3 where SINV is the dependent variable, difficulty but not demonstrability is a significant influence, supporting H1. Model 4 further supports the insignificance of demonstrability by replacing this variable with dummies for the knowledge and skill tasks, where judgment is the baseline. Neither of these coefficients is significant.

Models 5 and 6 examine effects on sender claims (SCLAIM). Again demonstrability is not significant. In support of H2, SINV is a significant explanator when added in model 6 . Senders are honest to the extent that their claims are tempered by what they really invested. Difficulty becomes insignificant when SINV is added because the influence of difficulty operates indirectly through sender confidence.

Models 7 to 11 focus on the major issue, the effect of difficulty and demon- 
strability on persuasion, proxied by RINV. ${ }^{6}$ The diagrams in figure 5 for PERS and RINV (bottom panel) suggest that persuasion falls with difficulty. They also suggest that persuasion falls when we compare skill with judgment, the highest and lowest levels of demonstrability. Averaged over all levels of difficulty, both average PERS and RINV are higher for skill (16.2 and 67.8) than judgment (10.4 and 62.0). To examine these effects we regress persuasion proxied by RINV on difficulty and demonstrability using BLIND RINV as a control for effects on receiver investment other than communication. Difficulty is negative and significant throughout: Harder tasks reduce persuasion. Model 7 shows that demonstrability is significant supporting H4.

While Laughlin and colleagues propose demonstrability changes along a spectrum (Laughlin and Hollingshead, 1995; Laughlin and Shupe, 1996), it is clear that such a spectrum, if it exists, would be ordinal rather than continuous in nature to the extent that skill, judgment and knowledge tasks are different in kind rather than merely in degree. We therefore examine whether the effect of demonstrability holds when we examine these three separately. In model 8 we again replace demonstrability with dummies for skill and knowledge. It reveals that beyond the lowest level of demonstrability (judgment), both skill and knowledge tasks raise persuasion. Further regressions (not reported) reveal that when one of these two lower demonstrability levels is used as a baseline, the other is insignificant suggesting there is no effect on persuasion as demonstrability is raised or lowered from skill to knowledge. Demonstrability therefore affects persuasion even if treated as a categorical variable.

We hypothesise that while demonstrability affects persuasion directly (H4), difficulty does so via the sender's message (H3). We examine this in models 9 to 11. SCLAIM is significant throughout, supporting H3. Difficulty remains significant, perhaps because this variable was communicated by senders in other ways rather than through claims about their own investments. Model 10 again replaces demonstrability with knowledge and skill, both of which are significant. ${ }^{7}$ In model 11 SINV is insignificant when added suggesting sender confidence variable does not have an effect on persuasion independently of SCLAIM. This suggests difficulty reduces persuasion mainly through senders' expressed messages rather than inability to persuade though lacking confidence. Social preferences and resulting lying costs provide a possible explanation.

\footnotetext{
${ }_{6}$ As a robustness test we re-estimated these models using PERS as the dependent variable without the control for BLIND RINV. The results we obtained were, in terms of variable significance, the same.

7 An $F$-test reveals no significant difference between the coefficients of knowledge and skill $(p=0.526)$.
} 


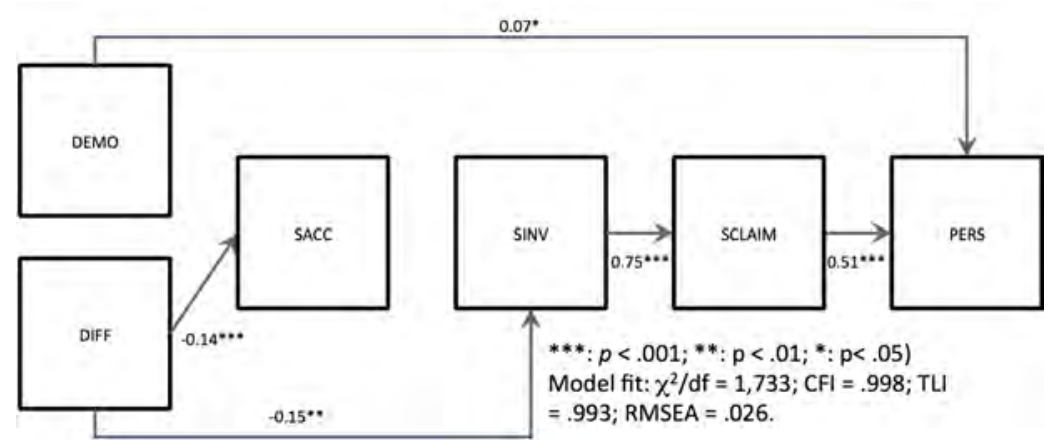

Fig. 6. Maximum likelihood estimation of the conceptual model.

\subsection{SEM Results}

In the interest of result robustness we also tested the hypothesised model of figure 3 by applying a covariance-based structural equation modelling approach (CB-SEM), using AMOS 24. The results are presented in figure 6. Again we use only data from phase $\mathrm{C}$ for all variables bar BLIND RINV.

The fit of our hypothesised model was excellent $\left(\chi^{2} / \mathrm{df}=1,733\right.$; confirmatory fit index $[\mathrm{CFI}]=.998$; Tucker-Lewis index $[\mathrm{TLI}]=.993$; root mean squared error of approximation $[\mathrm{RMSEA}]=.026)$. As for the structural model, the effect of sender's perceived difficulty on sender's investment (SINV) is negative and significant $(-.15, p<.01)$. However, the level of demonstrability does not affect SINV, thereby fully confirming H1. As for H2, we found that SINV positively and significantly relates to SCLAIM $(.75 ; p<.001)$, thereby indicating that senders are honest to the extent that their claims are tempered by what they really invested. In contrast and as expected, DEMO has no significant effect on SCLAIM, thereby fully confirming H2. In H3, we hypothesised that a sender's claim has a positive effect on the receiver's persuasion (PERS). The effect is positive, strong and significant $(.51 ; p<.001)$, thereby supporting H3. In addition, we also tested whether difficulty and SINV have direct effects on PERS, however, both relationships are non-significant. In conclusion, our results indicate that the negative effect of difficulty on PERS is fully mediated by both SINV and SCLAIM. In addition to difficulty, we hypothesised that demonstrability has a direct and positive effect of PERS. In accordance with $\mathrm{H} 4$, we find that demonstrability positively and significantly affects PERS $(.07 ; p<.05)$. As further hypothesised, the effect of demonstrability on SINV and SCLAIM is non-significant, thereby fully confirming H4. As pertaining to H5, we conducted a logistic regression, finding that more difficult images significantly cause less accurate sender decisions, while demonstrability had no effect on accuracy (SACC). These results fully confirm H5.

In conclusion, our empirical results from both types of analysis confirm all of our hypotheses. Overall they show that both task difficulty and demonstrability have an independent effect on the persuasion of the receiver of the message. The negative effect of difficulty on persuasion is fully mediated by the 
communication process between the sender and the receiver, while the effect of demonstrability relates directly and positively to sender's persuasion.

\section{Discussion}

Our results support the general idea that diverging interests impede the transmission of private information (Crawford and Sobel, 1982), an important source of market efficiency in the economy (Hayek, 1945). ${ }^{8}$ We contribute a further insight to this: Private information transmission depends on the problem solving that generated it. In our sender-receiver experiment, solution demonstrability and difficulty independently affect persuasion. Difficulty reduces receiver investment due to sender unwillingness to conceal it, thereby reducing their perceived expertise. Lacking demonstrability lowers receiver investments even when difficulty and sender confidence are controlled. Ceteris paribus, receivers are more cautious when decision problems lack objective success criteria. In contrast, under common knowledge of rationality, senders have no incentive to reveal difficulty, which affects solution accuracy. Rational receivers have none to act on lacking demonstrability, which does not.

One implication is that the information transmission problem is particularly true for industries where product performance is more subjective or difficult to ascertain. Another, more practical one is the existence of a curse of knowledge in selling: Senders tend to signal lacking expertise from decision difficulty that will negatively affect their persuasiveness (e.g. McGinnies and Ward, 1980).

We believe that this new perspective harbours potential for more insight into the relationship between advisor decision problems and persuasion. Future research could further develop the concept of demonstrability and vary it in more fine-grained experimental designs and explore how it interacts with other decision problem characteristics such as difficulty.

\footnotetext{
8 Our senders' overall accuracy of $78.9 \%$ means receivers (and therefore senders) could have made significantly higher gains had they invested more than their average $66.8 \%$ of points per game. Payoff-maximising, risk-neutral receivers should invest all 100 points if $\mathrm{SACC}>\frac{2}{3}$, i.e. if the marginal return of a point invested $(1.5 \times \mathrm{SACC})$ is greater than 1.
} 


\section{References}

Abeler, J., Becker, A., Falk, A., 2014. Representative evidence on lying costs. Journal of Public Economics 113, 96-104.

Ajzen, I., 2012. Attitudes and Persuasion. Oxford University Press, Ch. 15, pp. $367-393$.

Cai, H., Wang, J. T. Y., 2006. Overcommunication in strategic information transmission games. Games and Economic Behavior 56 (1), 7-36.

Camerer, C., Loewenstein, G., Weber, M., 1989. The curse of knowledge in economic settings: An experimental analysis. The Journal of Political Economy 97 (5), 1232-1254.

Carpentier, C., Suret, J.-M., 2015. Angel group members' decision process and rejection criteria: A longitudinal analysis. Journal of Business Venturing 30 (6), 808-821.

Chakraborty, A., Harbaugh, R., 2007. Comparative cheap talk. Journal of Economic Theory 132 (1), 70-94.

Chakraborty, A., Harbaugh, R., 2010. Persuasion by cheap talk. The American Economic Review 100 (5), 2361-2382.

Cialdini, 1988. Influence: Science and Practice. Scott, Foresman and Co., Glenview, IL.

Crawford, V. P., 1998. A Survey of Experiments on Communication via Cheap Talk. Journal of Economic Theory 78, 286-298.

Crawford, V. P., Sobel, J., 1982. Strategic information transmission. Econometrica, 1431-1451.

Croson, R., 2005. The method of experimental economics. International Negotiation 10, 131-148.

de Bettignies, J. E., Brander, J. A., 2007. Financing entrepreneurship: Bank finance versus venture capital. Journal of Business Venturing 22 (6), 808-832.

DeFillippi, R. J., Arthur, M. B., 1998. Paradox in project-based enterprise: the case of film making. California Management Review 40 (2), 125-139.

Dohmen, T., Falk, A., Huffman, D., Sunde, U., Schupp, J., Wagner, G. G., 2011. Individual risk attitudes: Measurement, determinants and behavioral consequences. Journal of the European Economic Association 9 (3), 522 550 .

Dulleck, U., Kerschbamer, R., Mar. 2006. On Doctors, Mechanics, and Computer Specialists: The Economics of Credence Goods. Journal of Economic Literature 44 (1), 5-42.

Emons, W., 1997. Credence goods and fraudulent experts. RAND Journal of Economics 28 (1), 107-119.

Farrell, J., Rabin, M., 1996. Cheap talk. The Journal of Economic Perspectives 10 (3), 103-118.

Fischbacher, U., 2007. z-Tree: Zurich toolbox for ready-made economic experiments. Experimental Economics 10 (2), 171-178.

Golosov, M., Skreta, V., Tsyvinski, A., Wilson, A., May 2014. Dynamic strategic information transmission. Journal of Economic Theory 151 (C), 304-341. 
Green, J. R., Stokey, N. L., 2007. A two-person game of information transmission. Journal of Economic Theory 135 (1), 90-104.

Hayek, F. A., 1945. The use of knowledge in society. The American Economic Review 35 (4), 519-530.

Herzenstein, M., Sonenshein, S., Dholakia, U. M., 2011. Tell me a good story and I may lend you money: The role of narratives in peer-to-peer lending decisions. Journal of Marketing Research 48, S138 - S149.

Janis, I. L., Hovland, C. I., Field, B. P., Linton, H., Graham, E., Cohen, A. R., Rife, D., Abelson, R. P., Lesser, G. S., King, B. T., 1959. Personality and Persuasibility. Yale University Press, New Haven, CN.

Jordan, J., McAuliffe, K., Rand, D., 2015. The effects of endowment size and strategy method on third party punishment. Experimental Economics 19 (4), 741-763.

Kirchsteiger, G., 1994. The role of envy in ultimatum games. Journal of Economic Behavior \& Organization 25, 373-389.

Laughlin, P. R., 1980. Social combination processes of cooperative problemsolving groups on verbal intellective tasks. In: Fishbein, M. (Ed.), Progress in Social Psychology. Vol. 1. L. Erlbaum Associates Inc., pp. 127-155.

Laughlin, P. R., 1999. Collective induction: Twelve postulates. Organizational Behavior and Human Decision Processes 80 (1), 50-69.

Laughlin, P. R., 2011. Group Problem Solving. Princeton University Press, Princeton, NJ.

Laughlin, P. R., Chandler, J. S., Shupe, E. I., Magley, V. J., Hulbert, L. G., 1995. Generality of a theory of collective induction: Face-to-face and computermediated interaction, amount of potential information, and group versus member choice of evidence. Organizational Behavior and Human Decision Processes 63 (1), 98-111.

Laughlin, P. R., Ellis, A. L., 1986. Demonstrability and social combination processes on mathematical intellective tasks. Journal of Experimental Social Psychology 22 (3), 177-189.

Laughlin, P. R., Hollingshead, A. B., 1995. A theory of collective induction. Organizational Behavior and Human Decision Processes 61 (1), 94-107.

Laughlin, P. R., Shupe, E. I., 1996. Intergroup collective induction. Organizational Behavior and Human Decision Processes 68 (1), 44-57.

Linzmayer, O. W., 2004. Apple confidential 2.0: The definitive history of the world's most colorful company. No Starch Press.

Lundquist, T., Ellingsen, T., Gribbe, E., Johannesson, M., 2009. The aversion to lying. Journal of Economic Behavior \& Organization 70 (1), 81-92.

Martens, M. L., Jennings, J. E., Devereaux, P., 2007. Do the stories they tell get them the money they need? the role of entrepreneurial narratives in resource acquisition. The Academy of Management Journal 50 (5), 1107-1132.

McGinnies, E., Ward, C. D., 1980. Better liked than right trustworthiness and expertise as factors in credibility. Personality and Social Psychology Bulletin $6(3), 467-472$.

O’Keefe, D. J., 2002. Persuasion: Theory and research. Sage Publications. 
Petty, R. E., Briñol, P., Tormala, Z. L., 2002. Thought confidence as a determinant of persuasion: the self-validation hypothesis. Journal of Personality and Social Psychology 82 (5), 722.

Petty, R. E., Wegener, D. T., Fabrigar, L. R., 1997. Attitudes and Attitude Change. Annual Review of Psychology 48, 609-647.

Pitchik, C., Schotter, A., 1987. Honesty in a model of strategic information transmission. The American Economic Review 77 (5), 1032-1036.

Pollack, J. M., Bosse, D. A., 2014. When do investors forgive entrepreneurs for lying? Journal of Business Venturing 29, 741-754.

Sah, S., Moore, D. A., MacCoun, R. J., 2013. Cheap talk and credibility: The consequences of confidence and accuracy on advisor credibility and persuasiveness. Organizational Behavior and Human Decision Processes 121 (2), 246-255.

Sánchez-Pagés, S., Vorsatz, M., 2007. An experimental study of truth-telling in a sender-receiver game. Games and Economic Behavior 61 (1), 86-112.

Stasser, G., Stewart, D., 1992. Discovery of hidden profiles by decision-making groups: Solving a problem versus making a judgment. Journal of Personality and Social Psychology 63 (3), 426-434.

Tenney, E. R., Spellman, B. A., MacCoun, R. J., 2008. The benefits of knowing what you know (and what you don't): How calibration affects credibility. Journal of Experimental Social Psychology 44 (5), 1368-1375.

van Werven, R., Bouwmeester, O., Cornelissen, J. P., 2015. The power of arguments: How entrepreneurs convince stakeholders of the legitimate distinctiveness of their ventures. Journal of Business Venturing 30 (4), 616-631.

van Winden, F., 1999. Experimental studies of signaling games. In: Luini, L. (Ed.), Uncertain Decision:s Bridging Theory and Experiments. Springer.

Wang, J. T.-y., Spezio, M., Camerer, C. F., 2010. Pinocchio's pupil: using eyetracking and pupil dilation to understand truth telling and deception in sender-receiver games. The American Economic Review 100 (3), 984-1007.

Wolinsky, A., 1993. Competition in a market for informed experts' services. The RAND Journal of Economics, 380-398.

Zimbardo, P. G., Leippe, M. R., 1991. The Psychology of attitude change and social influence. McGraw-Hill. 